\title{
Study of up-down poloidal density asymmetry of high-Z impurities with the new impurity version of XGCa
}

\author{
J. Dominski ${ }^{1} \dagger$, C.S. Chang ${ }^{1}$, R. Hager ${ }^{1}$, P. Helander ${ }^{2}$, S. $\mathbf{K u}^{1}$, and \\ E.S. Yoon ${ }^{3}$ \\ ${ }^{1}$ Princeton Plasma Physics Laboratory, 100 Stellarator rd, Princeton, NJ 08540, USA \\ ${ }^{2}$ Max-Planck-Institut für Plasmaphysik, Greifswald, Germany \\ ${ }^{3}$ Ulsan National Institute of Science and Technology, Ulsan 44919, South Korea
}

(Received $\mathrm{xx}$; revised $\mathrm{xx}$; accepted $\mathrm{xx}$ )

\begin{abstract}
Addition of multispecies impurity ions to the total-f gyrokinetic particle-in-cell code $\mathrm{XGCa}$ is reported, including a cross-verification of neoclassical physics against the NEO code. This new version of the neoclassical gyrokinetic code XGCa is used to benchmark and confirm the previous reduced-equation-based prediction that high- $\mathrm{Z}$ impurity particles in the Pfirsch-Schlüter regime can exhibit a significant level of up-down poloidal asymmetry, through the large parallel friction force, and thus influences the radial plasma transport significantly. The study is performed in a plasma with weak toroidal rotation. In comparison, when the impurity particles are in the plateau regime, the up-down poloidal asymmetry becomes weak, with the parallel friction force becoming weaker than the parallel viscous force. It is also found that the linearization of the perturbed distribution function, based on the small poloidal asymmetry assumption, can become invalid. Using the numerical data from XGCa, each terms in the parallel fluid force-balance equation have been analyzed to find that both the main ions and the electrons respond to the poloidal potential variation adiabatically when the high- $Z$ tungsten possesses large poloidal variation.
\end{abstract}

\section{Introduction}

Study of the high charge-number (high-Z) impurity particle effect in a tokamak plasma is a high priority research area especially because tungsten has been chosen as the armour material on the divertor plates. Experiments have found that the tungsten impurity particles alter the plasma confinement property significantly (Angioni et al. 2014; Loarte et al. 2015; Litaudon \& et al. 2017).

In this work, we report the extension of the total-f gyrokinetic code XGCa (Hager \& Chang 2016) to include multispecies gyrokinetic impurity particles. XGCa is the axisymmetric version of the XGC family of codes that utilize multispecies nonlinear Fokker-Planck collision operator (Hager et al. 2016). We also report verification of the new impurity feature in XGCa, against NEO (Belli \& Candy 2008, 2012). This multi-species collision operator is common to the family of XGC codes, such that this verification exercise is beneficial for other XGC versions. The new XGCa version is then used to study the neoclassical formation of up/down asymmetry in high-Z impurity particle density in a tokamak plasma with circular flux surfaces. Here, for simplicity, the high$\mathrm{Z}$ impurity species is chosen to be a fixed charge number tungsten. We note that the

$\dagger$ Email address for correspondence: jdominsk@pppl.gov 
previous gyrokinetic impurity transport study using XGC1 (Kim et al. 2017) in the core region of a tokamak plasma, with neoclassical and turbulence physics together, was performed in the older "full-f" version XGC1 that did not include kinetic electrons and did not use the recent "total-f" technique (Ku et al. 2018), in which the delta-f scheme is generalized to be mathematically equivalent to the usual full-f scheme. Our total-f scheme is briefly summarized in the next section for completeness.

This neoclassical work is to establish a baseline physics for future studies including neoclassical and turbulence physics together in complex edge geometry with X-point and divertor. First-principles based neoclassical tungsten physics in the core by itself is valuable because neoclassical physics could dominate the high-Z impurity transport in the core of tokamaks (Angioni et al. 2014) and because it interacts with turbulence (Kim et al. 2017; Estève et al. 2018) to self-organize. Moreover, understanding the poloidal asymmetries are important for tungsten particles as they can have a strong impact on their accumulation level in the burning core (Angioni \& Helander 2014; Angioni et al. 2015; Breton et al. 2018).

Poloidal asymmetries have already been studied theoretically and numerically. For instance, the poloidal variation of density due to friction force has been studied in references (Burrell \& Wong 1979; Tendler 1981), the increase of asymmetry amplitude with gradients or $Z$ was shown in equation (2) of reference (Brau et al. 1983), the role of impurity parallel friction forces in driving large amplitude asymmetries was shown by Chang \& Hazeltine (1980), and it was predicted analytically by Helander (1998) that parallel friction force dominates over parallel viscous force when impurities are in the Pfirsch-Schlüter (PS) regime, see discussion in chap. 2 of Reinke (2011). It was also discussed by Hirshman \& Sigmar (1976) that, at high collisionality, the Pfirsch-Schlüter flux (driven by the variation of the friction on a surface) dominates over the contribution of the local pressure anisotropy. Other early theoretical works by Chang (1983); Hinton \& Wong (1985); Romanelli \& Ottaviani (1998) have addressed the topic of asymmetries and in particular the role of centrifugal effects. In this paper, the considered plasma does not exhibit significant rotation. Therefore, the associated centrifugal effects, known to trigger tungsten accumulation on the outer midplane of tokamaks as shown in references (Fülöp \& Helander 1999; Reinke et al. 2013; Angioni et al. 2014, 2015; Köchl et al. 2018), are not studied here.

Compared to previous impurity-transport studies by other kinetic codes in the literature, XGC evolves the species total-f distribution functions and accounts for nonMaxwellian collisions between all species in five-dimensional phase space, thus providing the capability to resolve the formation of large amplitude poloidal asymmetries in gyrokinetic simulations.

In the XGCa simulations presented in this paper, which include almost no rotation, the parallel friction force between tungstens and the main deuterium ions become so large when $Z=40$ that: $1 /$ it dominates over the other drives (centrifugal force and parallel viscous force) of tungsten density's poloidal asymmetry, $2 /$ the use of a linearization of the distribution function can be invalid as $\delta f_{w} / f_{w} \sim 1$ (in agreement with (Angioni \& Helander 2014)), and 3 / it significantly affects the other species density asymmetries. Notably, both the electrons and main ions are found to respond adiabatically to this large tungsten asymmetry. In turn, the presence of tungsten impurities forces the main ion density asymmetry to be in the opposite direction than the one observed in the pure plasma simulation. Finally, we also show that when decreasing the tungsten charge from $Z=40$ to $Z=10$, the tungsten asymmetry decreases and is now essentially driven by the parallel viscous force. This dominant role of the parallel friction force over the parallel 
viscous force when the impurities are in the Pfirsch-Schlüter regime and the main ions in banana regime was predicted by Helander (1998).

The remainder of this paper is organized as follows. In section 2, we briefly present the XGCa model with multi-species gyrokinetic ions. In section 3, we present the test case plasma profiles and magnetic equilibrium we use in this paper, as well as, the different impure plasma species we simulate. In section 4, we present the verification exercise for the neoclassical impurity transport physics. In section 5, we show simulation results where large tungsten asymmetries arise. In section 6 , we show the role of parallel friction force in the formation of the large tungsten asymmetry. Finally, in section 7 a conclusion is drawn.

\section{XGCa model with impurities}

Even though most of the description given in this section is common to the total-f XGC family codes, we will use the name XGCa in this paper since XGCa is what is used in this study. XGCa is a total-f (Ku et al. 2018) gyrokinetic PIC code that is able to simulate large and small scale physics together. XGCa uses a hybrid-Lagrangian scheme in which the slow space-time varying component of the distribution function is gradually taken out from the particles and moved to a 5D phase-space grid, see (Ku et al. 2016, 2018). This technique permits to control the particle noise by mitigating the weightgrowth in the particle-part of the distribution function, $f_{p}$. The distribution function $f$ is composed of an analytic input part $f_{a}$, a grid part $f_{g}$, and a particle part $f_{p}$, such that $f=f^{a}+f^{g}+f^{p}$ : see the detailed discussion in (Ku et al. 2016, 2018).

The XGC family of codes, including XGCa, use the same new multi-species full$\mathrm{f}$ Fokker-Plank operator in Landau form evaluated on five-dimensional phase-space grid (Hager \& Chang 2016)

$$
\mathcal{C}_{s}=\sum_{s^{\prime}} \mathcal{C}\left[f_{s}, f_{s^{\prime}}\right]
$$

where the subscripts denote particle species. Collisions are computed between all pairs of species $\left(s, s^{\prime}\right)$ and evaluated with the total-f distribution function $f_{s}$. The complexity of the collision operator is quadratic with the number of species. This significantly increases the cost of simulations when many ion species are simulated.

In XGCa, the main and impurity ions are treated gyrokinetically. The only restriction in XGCa compared to the main version XGC (often called XGC1) is that in XGCa ("a" stands for axisymmetric) only the axisymmetric (toroidal Fourier mode number $n=0$ ) component of the electrostatic field is kept (Hager et al. 2016). Therefore, the grid is simplified and XGCa simulations are computationally less expensive than XGC1 simulations.

The gyrokinetic Poisson equation, $\mathcal{L} \phi=\bar{n}$, is solved when accounting for all ion species contributions

$$
\left(\sum_{s \in\{\text { ions }\}} \mathcal{L}_{s}\right) \phi=\sum_{s \in\{\text { ions }, \mathrm{e}-\}} \bar{n}_{s},
$$

with $\mathcal{L}_{s}$ the gyrokinetic polarization operator describing the polarization part of the ion species density. All ions, i.e., main and impurity, and electron contributions are accounted for. Electrons are drift-kinetic. See reference (Ku et al. 2018) for details about the original pure plasma Poisson solver which is implemented in XGC.

The same grid of gyroaveraging matrices is used for every ion species. Indeed, to compute the ion density, we first sum the gyrocenter charge density of all ion species on 

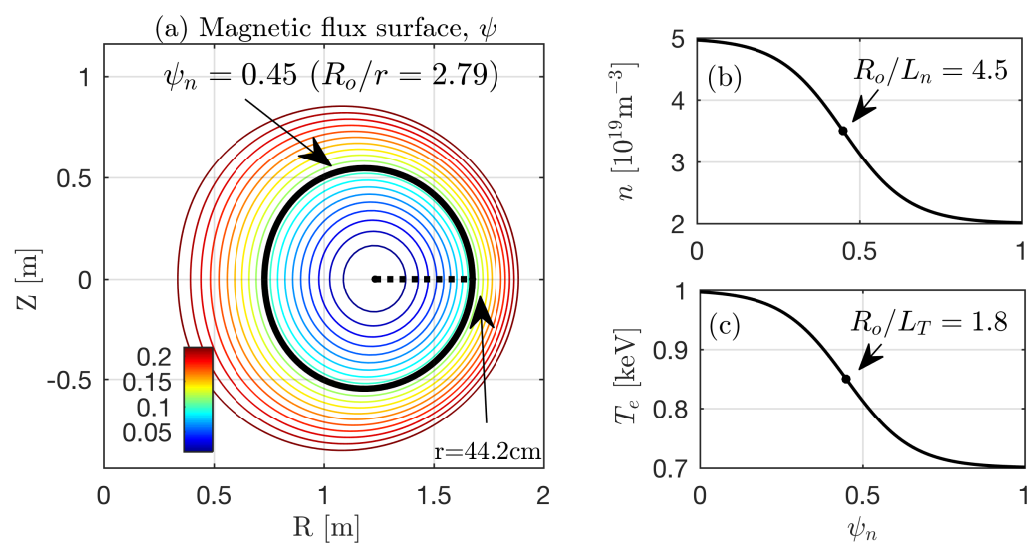

Figure 1. Test case used for this verification exercise. The magnetic flux surface are plotted in subplot (a) where the surface of interest $\psi_{n}=0.45$ is plotted with a thick black line. On this surface, $R_{o} / r=2.79$ is measured at outer most position of the surface where the minor radius is $r=0.442 \mathrm{~m}$. The density and temperature profiles are in (b) and (c), respectively. The value of the gradients are provided for the surface $\psi_{n}=0.45$ where these gradients are peaked and where we conduct our study. Note that $\psi_{n}=\psi / \psi_{\text {edge }}$. Gradients are estimated with $R_{o} / L_{n}=R_{o} / r_{o} d \ln n / d \sqrt{\psi_{n}}$ on the quasi-circular surface $\psi_{n}=0.45 . R_{o}=1.23 \mathrm{~m}$.

the same $4 \mathrm{D}$ grid by computing

$$
n(\boldsymbol{X}, \mu)=\sum_{s} Z_{s} \int d v_{\|} f_{s},
$$

and then we compute its gyroaverage to obtain the 3D charge density

$$
\bar{n}=\sum_{\mu}\langle n(\boldsymbol{X}, \mu)\rangle_{\alpha},
$$

with $\boldsymbol{X}=\boldsymbol{x}-\boldsymbol{\rho}$ the gyrocenter position, $\rho=\rho(\boldsymbol{X}, \mu, \alpha)$ the Larmor radius, $\boldsymbol{x}$ the particle position, $\mu$ the magnetic moment, $\alpha$ the gyrocenter, and \langle\rangle$_{\alpha}$ the gyroaveraging operation. More details on the gyroaveraging matrices are given in reference (Dominski et al. 2018). In case the Larmor radius differs significantly between ion species, it could be interesting to use an individual set of gyroaveraging matrices for each species, but this would require to gyroaverage the distribution function of each species separately thus increasing the computations. Implementation of such species-specific gyroaveraging matrices is left for future studies.

XGC can solve the whole plasma volume including the magnetic axis, the separatrix, and the open field line region of the scrape of layer (SOL). Neutral particles are recycled back to the plasma at the wall (Stotler et al. 2017), wall sheath is modeled with a logical sheath (Parker et al. 1993). Further details on the neutral particles and logical sheath can be found in reference (Ku et al. 2018). The present study does not include the open-field region, hence the logical sheath boundary condition and the neutral particle recycling are not used. Instead, we use Dirichlet boundary condition on the electrostatic potential $\phi$ at $\psi_{n}=1$.

\section{Test case description}

The considered test case has been introduced by Hager et al. (2019) for a pure plasma verification exercise between XGCa and NEO. It is a simple test case with almost no 


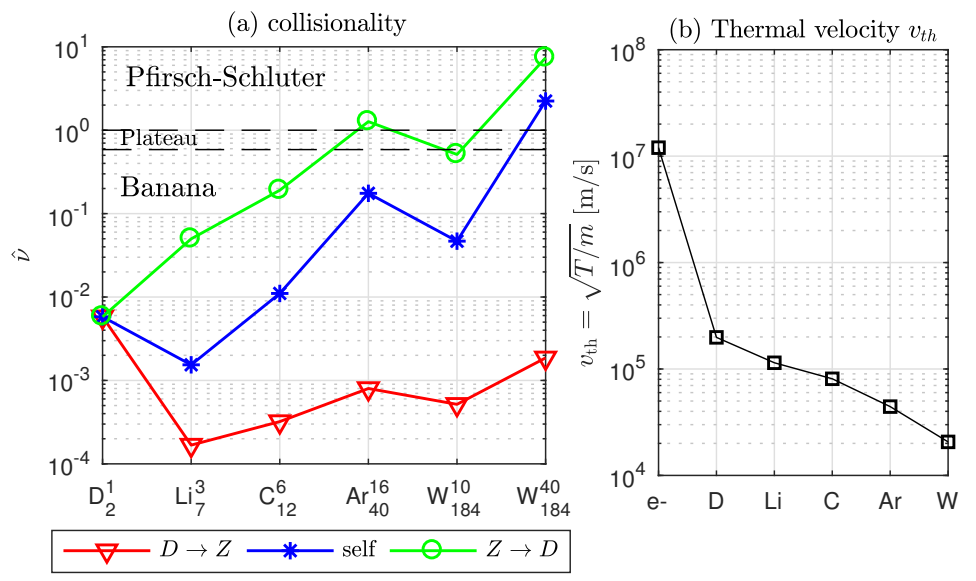

Figure 2. Collision regimes of the various ions. Subplot (a) Normalized collision time $\hat{\nu}=1 / \tau \omega_{t}$ of all ion species present in this paper simulations. The $\mathrm{x}$-axis represents the ion species, including deuterium ion and impurity ions. Self-collision $(Z \rightarrow Z)$ frequencies are in blue, collision frequencies of deuterium on the ions $(D \rightarrow Z)$ are in red, and collision frequencies of the ions on deuterium $(Z \rightarrow D)$ are in green. Subplot (b) is the thermal velocity of the different considered ions at surface $\psi_{n}=0.45$. Plasma parameters are taken at $\psi_{n}=0.45$, where $T=850 \mathrm{eV}$, $n_{e}=3.5 \times 10^{19}$, and $n_{D}=99 \% n_{e}$.

shaping or Shafranov shift, and tight aspect ratio, see figure 1 subplot (a). Magnetic equilibrium was built using FLOW (Guazzotto et al. 2004).

Physics of interest occurs at the magnetic surface $\psi_{n}=0.45$, where the gradients are peaked. This surface is plotted with a thick black line in subplot (a), where the gradients are peaked. On this surface, the local aspect ratio $R_{o} / r=2.79$ is close to typical value near the separatrix of conventional aspect ratio tokamaks. The main interest of having a large radial domain around the $\psi_{n}=0.45$ surface is to put the boundary $\psi_{n}=1$ away from the studied surface and avoid boundary effects. Simulation including x-point and more realistic boundary physics will be considered in following works. The profiles of density and temperature, respectively plotted in subplots (b) and (c), have mild gradients that peak at $\psi_{n}=0.45$ where $R_{o} / L_{n}=2.0$ and $R_{o} / L_{T}=0.81$. Deuterium is the main ion species with its physical mass and electric charge $(+e)$. The electrons are drift kinetic with their physical mass and electric charge $(-e)$. In this test case, collisions are weak for both electrons and ions (banana regime). All species are initialized with the same temperature $T_{e}=T_{D}$ (= $\left.T_{\text {impurities }}\right)$. The initial rotation is zero without a torque input. The fully developed impurity kinetic energy from toroidal rotation is $10 \%$ or less of the impurity pressure. In this paper, the diagnostics are measured at this surface $\psi_{n}=0.45$ where the gradients are peaked.

In this paper, multiple impure plasmas are considered. Each impure plasma contains a main ion (always deuterium) and a single impurity ion. Different single impurity ions have been considered: lithium (Li), carbon (C), argon (Ar), and tungsten (W). In all these impure plasma cases, the deuterium density is initialized to be equal to $99 \%$ of the electron density, $n_{D}=0.99 n_{e}$. The impurity-ion density is then chosen to ensure quasi-neutrality given the charge $Z$ of the impurity, $Z n_{Z}=0.01 n_{e}$. This small amount of impurity density is chosen to represent more realistic tungsten impurity physics. The charge of the different impurities is indicated in the x-axis of figure 2 subplot (a). Notably, two impure tungsten cases are considered: one with $Z=10$ and one with $Z=40$, because they are in different collision regimes. 

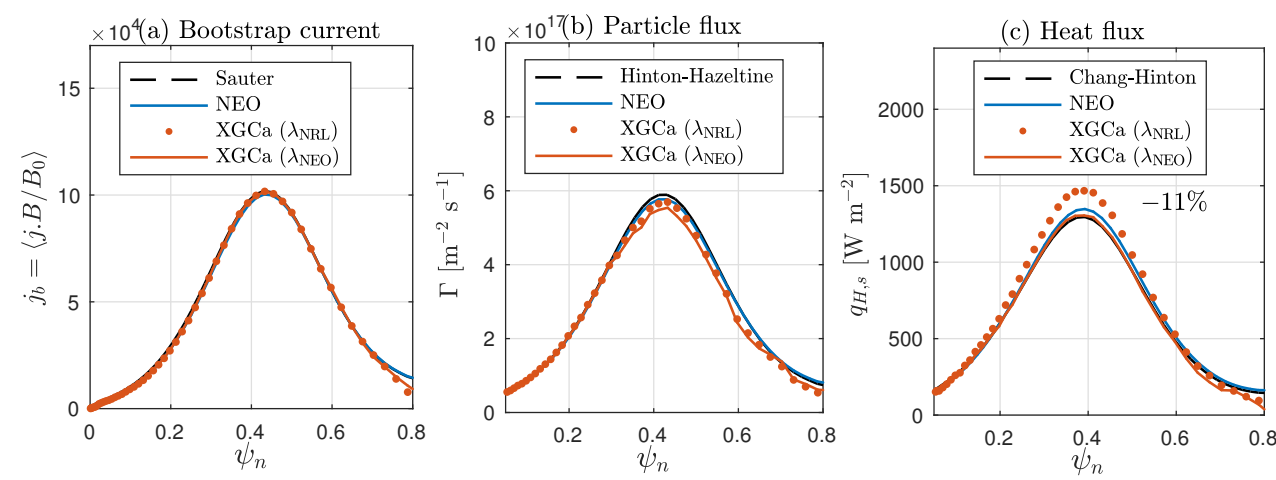

Figure 3. Verification of XGCa against analytical and NEO results, in the pure plasma case. XGC results have been produced with the usual Coulomb logarithm from the NRL formulary (Huba 2013), $\lambda_{\mathrm{NRL}}$, and with the Coulomb logarithm definition from NEO, $\lambda_{\mathrm{NEO}}$. Differences are small (up to $\sim 10 \%$ ) but the agreement is consistently improved when using the same definition. More details on the Coulomb logarithm are given in Hager et al. (2019).

The main-ion and impurity-ion collisionalities are computed for each impure plasma and plotted in figure 2. Collisionalities of $Z-Z$ (blue), $D-Z$ (red), and $Z-D$ (green) collisions have been computed using the normalized collision frequency

$$
\hat{\nu}_{i j}=\frac{1}{\tau_{i j} \omega_{t}} \text {. }
$$

This is the frequency of collision of species $i$ on target species $j$, and $\omega_{t}=\sqrt{T_{i} / m_{i}} / q R$ is the transit frequency. When $\hat{\nu}>1$ the species is in the Pfirsch-Schlüter regime, when $\hat{\nu}<\epsilon^{3 / 2}$ it is in the banana regime, else when $\epsilon^{3 / 2}<\hat{\nu}<1$ the species is in the plateau regime. The collision time of species $i$ on species $j$ is defined by

$$
\tau_{i j}=\frac{12 \pi^{3 / 2} \epsilon_{0}^{2}}{\sqrt{2}} \frac{\sqrt{m_{i}}}{q_{i}^{2} q_{j}^{2}} \frac{T_{i}^{3 / 2}}{n_{j} \ln \Lambda_{i j}},
$$

with $\ln \Lambda_{i j}$ the Coulomb logarithm given by Huba (2013) and $\tau_{i}=\sqrt{2} \tau_{i i}$. Discussion on the role of the Coulomb logarithm in inter-code comparisons can be found in Hager et al. (2019).

\section{Verification of neoclassical transport with the multi-ion-species version of $\mathrm{XGCa}$}

\subsection{Verification of a pure plasma with two deuterium species}

Prior to verify neoclassical physics in presence of an impurity, a verification of the new multi-ion-species implementation of XGCa has been made in a pure plasma simulation. To force the use of the new multi-ion-species functionality, we simply use two identical numerical species to model the deuterium ions. It's a very convenient first verification exercise. Results were obviously equivalent to the one obtained with a single deuterium species. These results are now cross-verified against NEO for the sake of completeness.

This first verification test case corresponds to the pure plasma verification exercise by Hager et al. (2019) where the single-ion-species version of XGCa was used. As mentioned before, we use two identical numerical species for deuterium (each numerical species represents $50 \%$ of the physical deuterium density) in order to force the use of the multi-ion-species implementation of XGCa. Results obtained with this multi-ion-species 

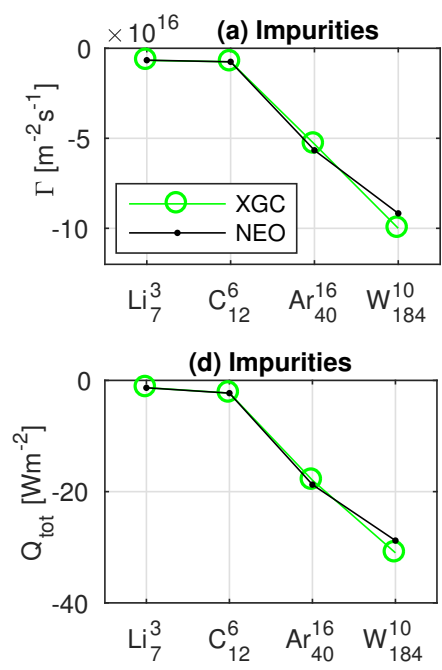

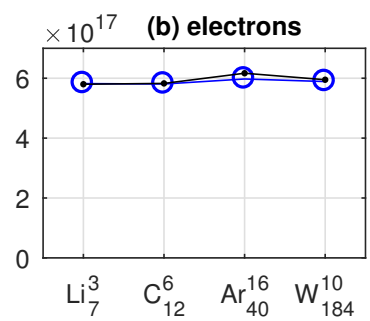

(e) electrons

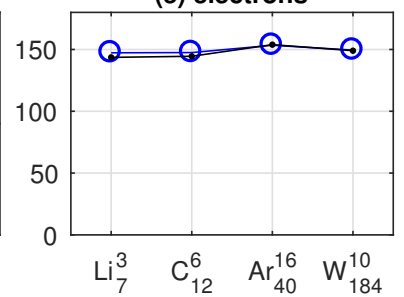

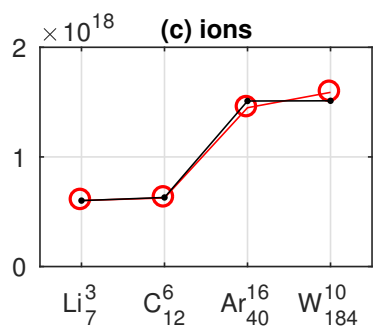

(f) ions

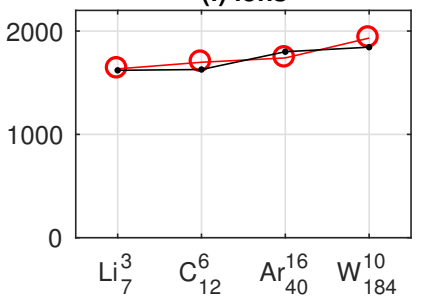

Figure 4. Neoclassical fluxes of particles (a,b,c) and energy (d,e,f) computed with XGCa (color and circle markers) are compared to NEO results (black dot markers). Differences are small (less than $8 \%$ ). The flux surface averaged fluxes are measured at $\psi_{n}=0.45$ where the gradients are peaked.

XGCa (red) are plotted in figure 3 and compared to both analytic predictions (black dashed) and NEO (Belli \& Candy 2008, 2012) simulation results (blue). In subplot (a), a very good agreement is found between the bootstrap current, $j_{\mathrm{b}}$, computed in XGCa, NEO, and with the Sauter formula (Sauter et al. 1999). In subplot (b), a very good agreement is found between the particle fluxes, $\Gamma$, computed with XGCa, NEO, and with the Hinton-Hazeltine formula (Hinton \& Hazeltine 1976). In subplot (c), a very good agreement is found between the heat fluxes computed with XGCa, NEO, and the ChangHinton formula (Chang \& Hinton 1982). The heat flux is defined by $q_{h}=Q_{\mathrm{tot}}-5 T \Gamma / 2$ with $Q_{\text {tot }}$ the kinetic energy flux and $\Gamma$ the particle flux.

As originally done by Hager et al. (2019), XGCa uses the same Coulomb logarithm, $\lambda_{\mathrm{NEO}}$, as the one implemented in NEO (Belli \& Candy 2012), for this benchmark only, in order to facilitate the verification exercise. Otherwise XGC uses the definition of the Coulomb logarithm, $\lambda_{\mathrm{NRL}}$, given in the NRL formulary (Huba 2013). As previously found by Hager et al. (2019) for this particular test case, a $\sim 10 \%$ difference is recovered in the ion heat flux obtained with these two different Coulomb logarithm. On the other hand, the particle and kinetic energy fluxes were less affected by the choice of the Coulomb logarithm for our test case.

\subsection{Verification of neoclassical transport in the presence of one impurity species}

Verification of various impure plasmas, in which the single-impurity charge density represents $1 \%$ of the electron's charge density (see section 3), are carried out. Four impure plasma simulations are cross-verified between XGC and NEO. The first one contains fully stripped Lithium ( $\mathrm{Li}$ ) in the banana regime, the second one contains fully stripped Carbon (C) in the banana regime, the third one contains partly ionized Argon (Ar), $Z_{A r}=16$, in the Pfirsch-Schlüter regime, and the fourth one contains partly ionized Tungsten (W), $Z_{W}=10$, in the plateau regime. Collision frequencies are shown in figure 2 and discussed in the text.

Results are plotted in figure 4, where the particle flux (first row) and energy flux 
(second row) are measured at surface $\psi_{n}=0.45$ and plotted for each species: impurities in the first column, electrons in the second column, and main deuterium ions in the third column. In each subplot, the results of the four impure plasma cases simulated with $\mathrm{XGCa}$ (color circles) and NEO (black dots) are compared. The agreement is very good with a few percent difference (2 to $8 \%$ ).

The impurity particle fluxes of Argon and Tungsten are significantly larger than the one of Carbon and Lithium, despite that the density of Argon and Tungsten are the smallest (the charge density of impurity species represents $1 \%$ of the electron charge: $n_{Z}=0.01 n_{e} / Z$ for an impurity of charge $e Z$ ). The main ion (D) is found to compensate for the large impurity inward particle flux. The outward deuterium particle flux significantly increases in presence of argon or tungsten. As we will see in section 5, the particle flux of deuterium is even more affected by the tungsten $Z=40$. On the other hand, the electrons are slightly affected by the collision with the impurity ions. As expected, in both NEO and XGC, the electron flux is more significantly affected by collisions with argon impurities which have the highest $Z$ in this verification scan. As we will see in simulations of section 5 , the electrons are even more affected by collisions with tungsten $Z=40$.

\section{Large amplitude tungsten asymmetries}

Poloidal asymmetry of large amplitude, $\delta n /\langle n\rangle \simeq 40 \%$, is observed for the highly collisional tungsten with $Z=40$ in figure 5 . When we decrease the tungsten's electric charge from $Z=40$ to $Z=10$, we observe that the up/down asymmetry decreases by a factor $\sim 5$, as shown in figure 5 (a). To assess the convergence of these asymmetries measurements, their time evolution is plotted in figure 11 of the appendix. The up-down asymmetry plotted in figures 5 and 11 are measured with an estimate defined by

$$
\Upsilon_{w}=\frac{1}{\mathcal{N}} \oint d \theta \sigma\left(n_{w}-\left\langle n_{w}\right\rangle\right) /\left\langle n_{w}\right\rangle
$$

with $\sigma=+1$ on the upper part of the poloidal plane and $\sigma=-1$ on its lower part. The normalization factor is set to $\mathcal{N}=\oint d \theta \sigma \sin \theta \simeq 0.64$. Formally, the asymmetry of a field $\mathcal{A}$ is measured by $\Upsilon[\sigma, \mathcal{A}](t)=\oint d \theta(\sigma \mathcal{A}(t)-\langle\mathcal{A}(t)\rangle) /|\langle\mathcal{A}(t)\rangle| / \mathcal{N}$ where one uses $\sigma=H\left(z-z_{\text {axis }}\right)-H\left(z_{\text {axis }}-z\right)$ to measure the up-down asymmetry and $\sigma=$ $H\left(r-r_{\text {axis }}\right)-H\left(r_{\text {axis }}-r\right)$ to measure the in-out asymmetry. $H$ is the Heaviside step function, $r$ is the horizontal position, $z$ is the vertical position, and $\left(r_{\text {axis }}, z_{\text {axis }}\right)$ is the position of the magnetic axis.

Figure 5 clearly shows that the presence of tungsten impurities triggers the formation of asymmetries. Moreover, the up/down asymmetries have significantly larger amplitude than the in/out asymmetries. Note that we verified, that $Z=40$ tungsten asymmetries with same orientation and similar relative amplitude $\left(\delta n_{w} / n_{w}\right)$ are observed in a simulation with 400 times less tungsten, i.e., $n_{w}=0.01 n_{e} / Z / 400=2.5^{-5} n_{e} / Z$.

In/out asymmetries are weaker than up/down ones, but it is interesting to see that they are significantly different between $Z=10$ and $Z=40$. Indeed, the tungsten asymmetries are of opposite phase in these two cases. When $Z=10$ the tungsten weakly accumulates outwardly, whereas when $Z=40$ tungsten more significantly accumulates inwardly. The weak outward accumulation of tungsten in the $Z=10$ case is likely the consequence of the weak plasma rotation and the corresponding centrifugal force (no source of rotation is imposed in our plasma, but the kinetic energy in the tungsten rotation is $\sim 10 \%$ of the tungsten thermal energy). Electrons are then neutralizing the tungsten charge asymmetry and their adiabatic response explains the potential asymmetry. On the other hand, in 

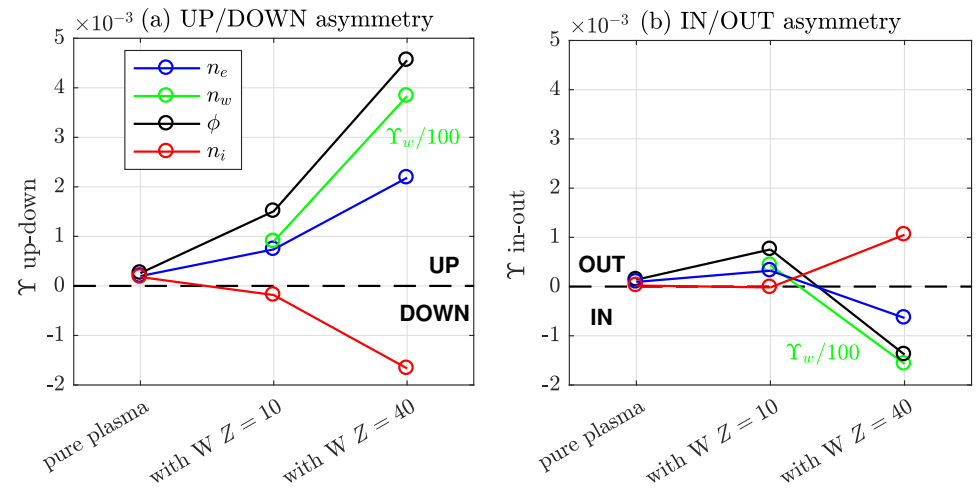

Figure 5. Up-down (a) and in-out (b) asymmetries of the electrostatic potential(black), the electron density (blue), the main deuterium ion (red), and the tungsten impurity (green) are measured with equation (5.1). Tungsten asymmetries measurement are divided by 100 to fit in the plot, but we remind the reader that tungsten represents $1 / 100$ of the electron charge, such that $0.01 \Upsilon_{w} \simeq \Upsilon_{e}-0.99 \Upsilon_{D}$ as one can verify on this figure. Each subplot contains the measurement for three simulations reported on the $\mathrm{x}$-axis: pure plasma, the impure plasma with tungsten $Z=10$, and the impure plasma with tungsten $Z=40$. Measurements are made at surface $\psi_{n}=0.45$.

the plasma with tungsten $Z=40$, the friction force between tungsten and the main ions are so strong that it overpowers the centrifugal force, as we will show in section 6 . In this case, the friction force dominates and the in-out asymmetry does not have to follow the centrifugal force direction.

Moreover, in the plasma with tungsten $Z=40$, the effect from tungsten charge density is so strong that both deuterium and electron species respond almost adiabatically (see figure 9), thus explaining their in/out asymmetry opposite to the tungsten's one. In particular, the deuterium up/down asymmetry is larger by a factor $\sim 10$ when $Z=40$ compared to when $Z=10$, which is interesting because when $Z=40$ the density of tungsten is 4 times smaller but its effect on the deuterium up/down asymmetry is 10 times bigger.

The fact that deuterium and electron particles requires stronger adiabatic response to neutralize the large amplitude asymmetry of collisional tungsten particles is interesting, because it suggests that the high-Z tungsten could interact with both electron and ion non-adiabatic responses to the turbulent electrostatic fluctuations. This study is left for a future investigation.

These big amplitude asymmetries of tungsten density have a strong impact on the tungsten fluxes (Angioni \& Helander 2014) and must be properly resolved to calculate the neoclassical transport. To highlight the importance of solving the large amplitude asymmetries, we carried out NEO (Belli \& Candy 2008, 2012) simulations that utilizes linearization of the distribution function $f \simeq f_{0}+\epsilon f_{1}$ with small $\epsilon$ assumption, where $f_{0}$ is a flux function. As expected, the results obtained from the linearized code disagree from the total-f XGCa results when large asymmetries develop: see the $Z=40$ simulation in figure 6. In comparison, when asymmetries have small amplitudes $|\tilde{n} /\langle n\rangle| \lesssim 10 \%$ the results obtained with the small ordering approximation are in good agreement with XGCa, showing less than $10 \%$ difference in the $Z=10$ case. We find that when the collision frequency is high and the asymmetry is large $(Z=40)$, the local tungsten distribution function is nearly Maxwellian at all positions. There is essentially a shift in the $v_{\|}$direction, $f \propto \exp \left(m\left(v_{\|}-U_{\|}\right)^{2} / 2 T\right)$. 
(a) Tungsten

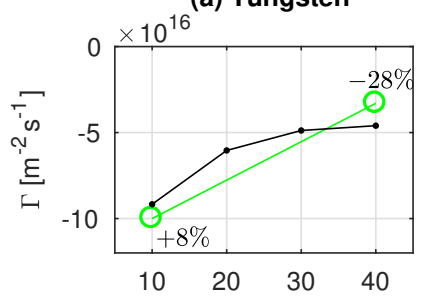

(d) Tungsten

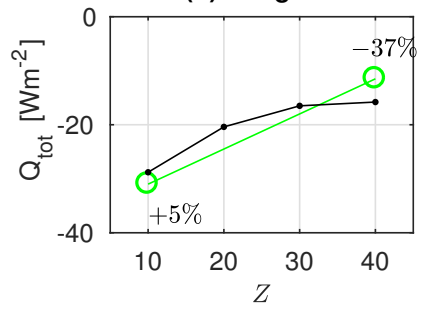

(b) electrons

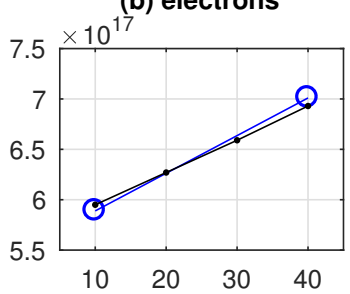

(e) electrons

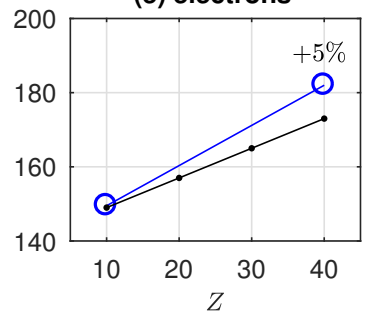

(c) Deuterium

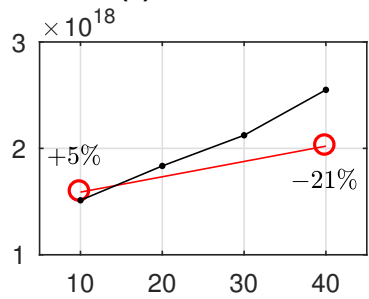

(f) Deuterium

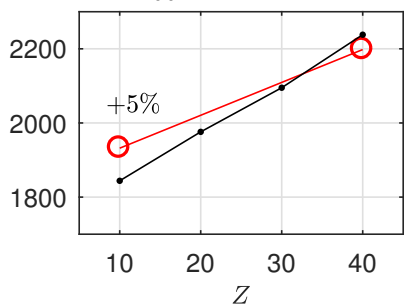

Figure 6. Neoclassical fluxes of particle (a,b,c) and energy (d,e,f) measured at the peak of gradient. The plasma contains tungsten which charge represents $1 \%$ of the electron charge. Results obtained using a linearization of the distribution function $f=f_{0}+\epsilon f_{1}$ are in black with dot markers and results obtained with XGCa are in color with circle markers. Diagnostics are measured at $\psi_{n}=0.45$ where the gradients are peaked.

The deuterium neoclassical fluxes can be affected by the presence of impurities through cross-species collisions or through the quasi-neutrality condition that enforces $\Gamma_{D}=$ $\Gamma_{e}-Z_{W} \Gamma_{W}$. Indeed, when the asymmetries are large and the tungsten particle flux is significant $\left|Z_{W} \Gamma_{W} / \Gamma_{e}\right| \simeq 1$, the deuterium particle flux is significantly affected by the presence of impurities. In case of $Z=40$, when tungsten asymmetries of large amplitude have developed, the tungsten particle flux is underestimated by the small ordering approximation $f(\psi, \theta) \simeq f_{0}(\psi)+\epsilon f_{1}(\psi, \theta)$ and the main ion particle flux is overestimated. We note here that, from figure $6(\mathrm{a}-\mathrm{c})$, the ambipolar particle flux is roughly maintained between impurity $\left(Z_{W} \Gamma_{W}\right)$ and main ion $\left(\Gamma_{D}\right)$ as usually assumed in analytic theories. However, the electron particle flux is not negligibly small in both NEO and XGCa. Let us also point out that, we also verified that the $Z=40$ tungsten particle flux, caused by the friction on the main ion, is proportional to the tungsten density. For this purpose, we have run a simulation that uses a tungsten density 400 times smaller than th eone of our reference test case, and we measured the tungsten particle flux to decreases by the same factor 400. In this particular case the tungsten charge flux is so small that the main ions (D) particle flux is not significantly affected by the tungsten. Finally, the electron fluxes are in good agreement between NEO and XGCa in figure 6 (b,e), even for the simulation with $Z=40$ where a large tungsten poloidal asymmetry is present. It shows that, in our test case problem, the fluxes of electrons are not strongly affected by the presence of tungsten's poloidal asymmetry (they respond adiabatically to this large poloidal asymmetry). The slight increase $(\sim+20 \%)$ of the electron fluxes observed when increasing the charge from $Z=10$ to $Z=40$ is rather due to the stronger electron collisions with $Z=40$ tungsten than with $Z=10$ tungsten. Indeed, collisions of electrons with tungsten are proportional to $\nu_{e, w} \propto Z_{w}^{2} n_{w}$ such that in our scan where we keep the tungsten charge density fixed $\left(n_{w}=n_{e} / 100 / Z_{w}\right)$ the collision $\nu_{e, w}$ will increase "proportionally" with $Z_{w}$. 


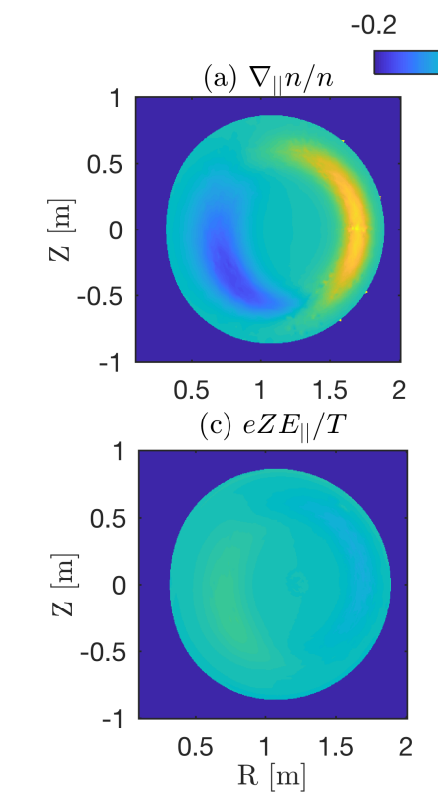

0.2
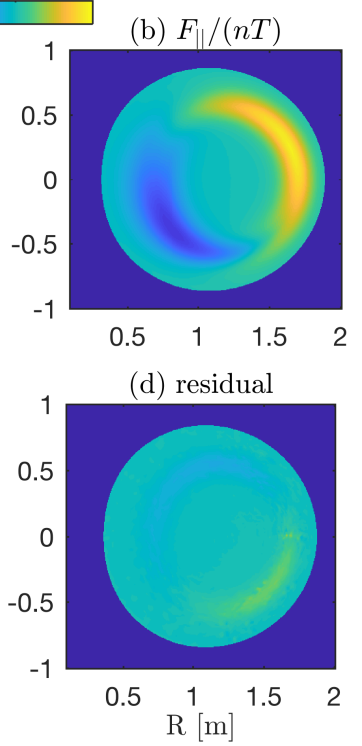

Figure 7. Components of the reduced parallel momentum equation (6.2), for simulation with tungsten $Z=40$, normalized in the following form: $\nabla_{\|} \ln n=Z e E_{\|} / T+F_{\|} / n T+$ residual, where each term is in units of $m^{-1}$. The residual is the remaining part of the density gradient not driven by electric field and friction force.

\section{Drive for asymmetries}

Physics of asymmetry formation can be understood from the parallel momentum equation

$$
m_{a} n_{a} \frac{d U_{\|, a}}{d t}=-\nabla_{\|} p_{a}-\mathbf{b} \cdot \nabla \cdot \pi_{a}+e_{a} n_{a} E_{\|}+F_{\|, a}
$$

with the fluid moments defined in appendix A. In our case, the parallel friction force $F_{\|}$is found to be the drive for the large poloidal asymmetry of tungsten with $Z=40$, as shown in figure 7. This is in agreement with Helander (1998), where it was shown that in the case where main ions are in the banana regime and the impurities are in the Pfirsch-Schlüter regime, the parallel viscous force can be neglected and the parallel momentum equation for tungsten reduces to, with a small residual term,

$$
\nabla_{\|} \ln n_{w}=e Z E_{\|} / T_{w}+F_{\|, w} / n_{w} T_{w} \quad \text { (+residual) }
$$

where "residual" represents the rest of the terms in equation (6.1) and is mostly from the parallel viscous force in a near steady-state. From the XGCa simulation data, we can verify this reduced equation and gain more insight on the physics at play.

The different components of equation (6.2) that drive the species density asymmetry on the surface $\psi_{n}=0.45$ are plotted in figure 8 for each species of three different simulations. Results of the impure plasma simulation with tungsten $Z=40$ are in the first column (left); results with tungsten $Z=10$ are in the second column (middle); and the results of the pure plasma simulation are in the third column (right). The parallel momentum equation (6.2) is analyzed for each species separately: tungsten results are in the first row, the deuterium results are in the second row, and the electron results are in the last row. For example, subplot (a) contains the different terms of the tungsten's parallel momentum equation (6.2) obtained from the XGCa simulation with tungsten impurity 

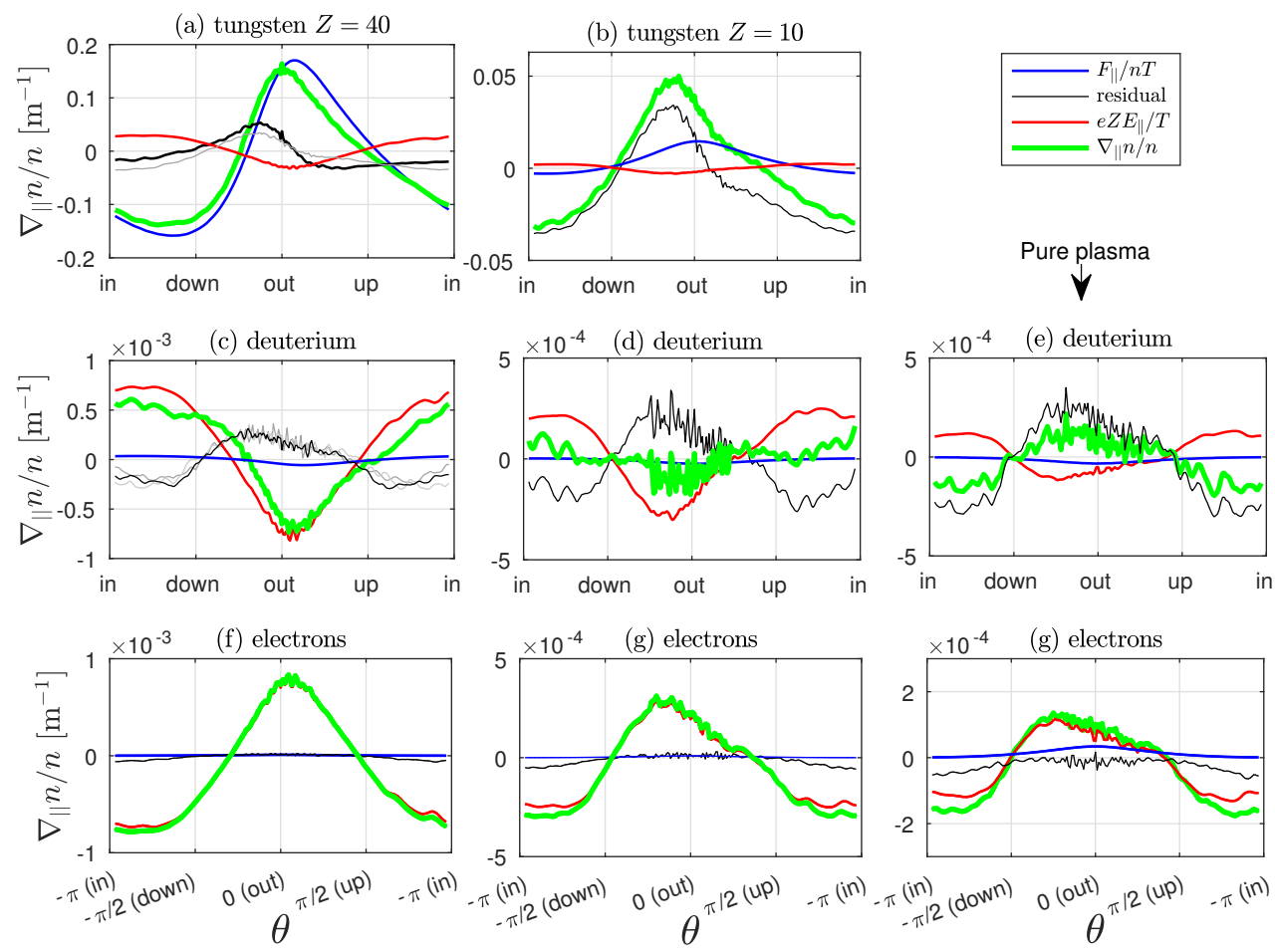

Figure 8. Drive of density asymmetry on the surface $\psi_{n}=0.45$ along the poloidal direction $\theta$, for three simulations: impure plasma with tungsten $Z=40$ (first column), impure plasma with tungsten $Z=10$ (second column), and pure plasma (last column). In each subplot, the different components of the species parallel momentum equation (6.2) are shown parallel friction force in blue, parallel electric field in red, parallel density gradient in green, and residual containing the parallel viscous force in black. Grey lines in subplots (a) and (b) are the residuals from other simulations (b), (d), and (e) for comparison purpose.

at $Z=40$, and subplot (c) contains the terms of the equation (6.2) for deuterium in the same impure simulation.

It clearly appears, in the last row of this figure 8, that electrons always respond adiabatically to the parallel electric field and that other terms contributing to the asymmetry are mainly negligible. Indeed, the electric field term (red) is almost equal to the density gradient (green). It also appears that the amplitude of the parallel electric field asymmetry (red) increases with the asymmetry of the tungsten density. This can be understood by the fact that more electrons are needed to neutralize the bigger tungsten asymmetry. Therefore, electrons having a strong adiabatic response, a strong asymmetry of the electrostatic potential develops.

A consequence of the increase of the parallel electric field is that the deuterium asymmetry becomes dominated by its adiabatic response to the parallel electric field in the impure plasma with tungsten $Z=40$ (figure 8 (c)) rather than by the parallel viscous force in the pure plasma (figure 8 (e)). In subplot (c), the electric field term (red) is almost equal to the density gradient (green), whereas in subplot (e) the residual containing the parallel viscous force (black) is almost equal to the density gradient (green). This increase of the adiabatic response further causes a reversal of the deuterium asymmetries, from an accumulation at top in the pure plasma simulation to an accumulation at bottom in the impure plasma with tungsten $Z=40$, as shown in previous figure 5 (a). In case of 


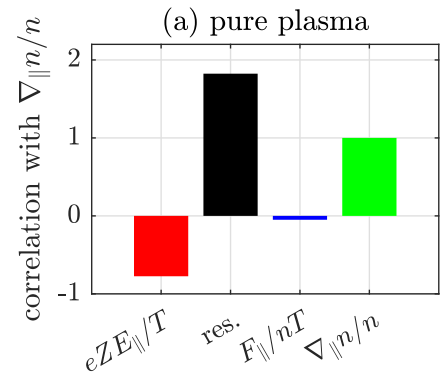

(b) impure plasma $Z=10$

(c) impure plasma $Z=40$
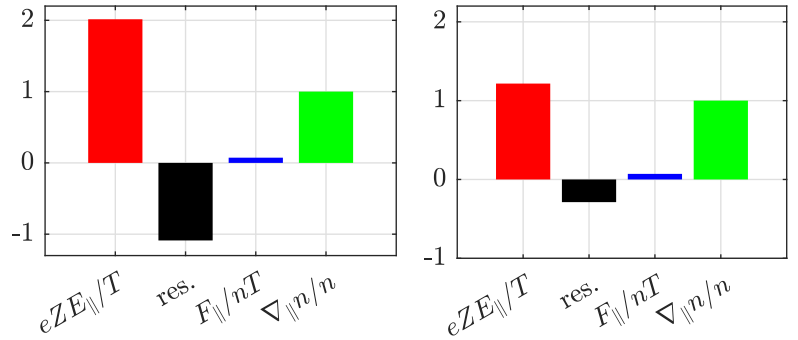

FIGURE 9. Deuterium's parallel momentum equation. More particularly, the contribution of each term of equation (6.2) to the deuterium parallel density gradient. Each component's contribution is estimated by its correlation (Eq. (6.3)) with the deuterium parallel gradient $L_{\|, d}^{-1}=\nabla_{\|} n_{d} / n_{d}$. Simulations of the (a) pure plasma, (b) impure plasma with tungsten $Z=10$, and (c) impure plasma with tungsten $Z=40$. Diagnostics made at surface $\psi_{n}=0.45$ with data of figure 8 subplots (c), (d), and (e).

the impure plasma with $Z=10$ the parallel electric field and the parallel viscous force nearly cancel each others in the deuterium parallel momentum equation $(\operatorname{subplot}(d))$, such that the deuterium asymmetry is weak for this value of $Z$ as previously shown in figure 5 .

This increase of the adiabatic response of deuterium associated to a reversal of the deuterium density asymmetry is illustrated in figure 9 , where we plot the correlation

$$
\operatorname{corr}\left[\mathcal{A}, L_{\|}^{-1}\right]=\frac{\int d \theta \mathcal{A}(\theta) L_{\|}^{-1}(\theta)}{\int d \theta L_{\|}^{-1}(\theta) L_{\|}^{-1}(\theta)},
$$

between the parallel gradient $L_{\|}^{-1}=\nabla_{\|} n / n$ and each term $(\mathcal{A})$ on the right-hand-side of equation (6.2). In case of the pure plasma in subplot (a), the deuterium asymmetry is driven by the residual as shown by its positive correlation with the parallel deuterium density gradient $L_{\|, D}^{-1}$. The parallel electric field term is anti-correlated to $L_{\|, D}^{-1}$ for this pure plasma. In comparison, for plasmas containing tungsten impurities, the deuterium asymmetry is driven by the parallel electric field as shown by its positive correlation with $L_{\|, D}^{-1}$ plotted in subplots (b) and (c), where $\operatorname{corr}\left[e Z E_{\|} / T, L_{\|}^{-1}\right] \simeq 2$ with $Z=10$ and $\simeq 1$ with $Z=40$, respectively. The correlation converging towards unity proves that the response of deuterium is mainly adiabatic when $Z=40$.

Finally, we can see that the tungsten density asymmetry is driven by the parallel friction force when $Z=40$ (figure 8 (a)) and by the parallel viscous force when $Z=10$ (figure 8 (b)). With the highly collisional tungsten $(Z=40)$, we can even further narrow down the parallel momentum equation to be, at leading order,

$$
\nabla_{\|} \ln n_{w} \simeq F_{\|, w} / n T_{w} .
$$

This observation is confirmed in figure 10 where we compute the correlations between the terms on the right-hand-side of the parallel momentum equation (6.2) with the tungsten parallel gradient term $\left(L_{\|, w}^{-1}=\nabla_{\|} n_{w} / n_{w}\right)$ on the left-hand-side. Given that tungsten collisionality is at banana-plateau level for $Z=10$ and at Pfirsch-Schlüter level for $Z=$ 40 , it shows the transition from a low collisional regime where the tungsten asymmetries are driven by parallel viscous force towards a high collisional regime where the tungsten asymmetries are driven by parallel friction, as predicted by Helander (1998). See collision frequencies plotted in figure 2 . 


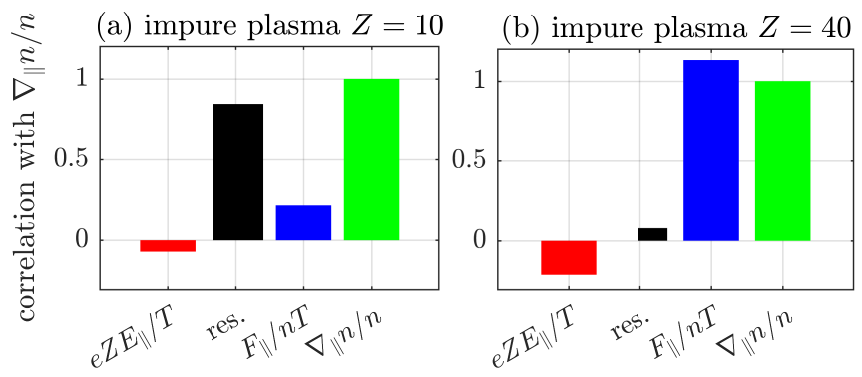

Figure 10. Tungsten's parallel momentum equation. More particularly, the contribution of each term of equation (6.2) to the tungsten parallel density gradient. Same calculation than in figure 10 but for tungsten. Subplot (a) and (b) show the results form the impure plasma simulations with (a) tungsten $Z=10$ and (b) tungsten $Z=40$. Diagnostics made at surface $\psi_{n}=0.45$ with data of figure 8 subplots (a) and (b).

\section{Conclusion}

Neoclassical gyrokinetic simulations of high-Z impurity transport in a tokamak geometry have been carried-out with the new multi-ion-species version of XGCa, which uses a multi-species full-f non-Maxwellian Fokker-Planck collision operator. Successful verification of the new impurity feature of XGCa against NEO has been carried out in NEO's validity regime.

The role of the parallel friction force on the formation of large poloidal asymmetries of high-Z tungsten (up to $\delta n_{w} / n_{w} \simeq 40 \%$ for $Z=40$ ) has been shown. In comparison, asymmetries of smaller amplitude $(\simeq 10 \%)$ for $Z=10$ tungsten in the plateau regime are driven by the parallel viscous force. These numerical results confirm the theoretical prediction by Helander (1998) that the parallel friction force dominates over the parallel viscous force when impurities are in the Pfirsch-Schlüter regime and the main-ions in the banana regime.

The usual small perturbation ordering $f=f_{0}+\epsilon f_{1}$ with $f_{0}$ a flux surface quantity $\left(f_{0}=f_{0}(\psi)\right)$ is found inaccurate in the Pfirsch-Schlüter collisional tungsten case where large amplitude poloidal asymmetries arise. Nonetheless, the $Z=40$ tungsten remains near local Maxwellian, such that poloidal density asymmetries could be prescribed in $f_{0}$ in order to keep $f_{1}$ small.

The in/out poloidal asymmetries of tungsten density are not negligible, even if they are found to be weaker than up/down asymmetries. The in/out asymmetry of tungsten are found to have an opposite direction at high-Z $(Z=40)$ compared to the one observed at low-Z $(Z=10)$. Indeed, when $Z=40$ the friction force overpowers all other drives of in/out asymmetry. As a consequence, the tungsten accumulates inwardly for this case with $Z=40$. In comparison, when $Z=10$, the friction force is weaker and some small centrifugal effects push the $Z=10$ tungsten to accumulate outwardly. The plasma we studied has very weak rotation. This nonetheless highlights the possible competition between the friction force and the centrifugal force to drive an in/out asymmetry of opposite direction.

The main ion (D) poloidal asymmetry is found, in our simulations, to be reversed by the formation of the large poloidal asymmetry of tungsten density. This reversal of the deuterium asymmetry is found to be caused by the deuterium adiabatic response to the parallel electric field, which increases with the increase of the electric field asymmetry in presence of tungsten. As a consequence of the main ions' enhanced adiabatic response, the main ion $(\mathrm{D})$ up/down asymmetry is measured to be $\sim 10$ times larger when $Z=40$ 
than when $Z=10$. This is interesting because, in our simulations, when $Z=40$ the density of tungsten is 4 times smaller but its effect on the deuterium is 10 times bigger.

Both the main ion and electron asymmetries are thus found to be dominated by their adiabatic response when large poloidal asymmetries are present on high-Z impurities. This suggests that high-Z tungsten particles can significantly interact with the nonadiabatic responses of electrons and main ions. In particular, the interaction of tungsten asymmetries with both non-adiabatic ion an electron responses to micro-turbulence fluctuations is left for a future study.

Following the successful verification and benchmark of neoclassical single impurity species transport physics, XGCa is currently being used for studying the transport of multi-charge state tungsten in JET ITER-like-Wall plasmas. In particular, the physics of tungsten transport in the pedestal with strong poloidal asymmetries is studied. Atomic interactions (ionization/recombination of tungsten) between different tungsten charge states are also being implemented in XGC. The results of these studies will be presented in the near future.

\section{Acknowledgments}

The authors would like to thank R.M. Churchill and M.J. Cole for helpful discussions. Funding has been provided via the SciDAC-4 program, Partnership Center for Edge Plasma Simulation (EPSi). Computing resources was provided by the National Energy Research Scientific Computing Center (NERSC) that operates under the DOE contract DE-AC02-05CH11231.

Notice: This manuscript has been authored by Princeton University under US Department of Energy Contract No. DE-AC02-09CH11466. The publisher, by accepting the article for publication, acknowledges that the United States Government retains a nonexclusive, paid-up, irrevocable, world-wide license to publish or reproduce the published form of this manuscript, or allow others to do so, for United States Government purposes. 


\section{Appendix A. Fluid moments}

$$
\begin{aligned}
U_{\|, a} & =\mathbf{b} \cdot \mathbf{U}_{a} \\
p_{a} & =n_{a}\left(2 T_{\perp, a}+T_{\|, a}\right) / 3 \\
n_{a} & =\int d \mathbf{v} f_{a} \\
T_{\|, a} & =\int d \mathbf{v} m_{a}\left(v_{\|}-U_{\|, a}\right)^{2} f_{a} / n_{a} \\
T_{\perp, a} & =\int d \mathbf{v}\left(m_{a} v_{\perp}^{2} / 2\right) f_{a} / n_{a} \\
\mathbf{U}_{a} & =\int d \mathbf{v} \mathbf{v} f_{a} / n_{a} \\
\Pi_{a} & =\int d \mathbf{v} m_{a} \mathbf{v} \mathbf{v} f_{a} \\
\pi_{a} & =\int d \mathbf{v} m_{a}\left[\left(\mathbf{v}-\mathbf{U}_{a}\right)\left(\mathbf{v}-\mathbf{U}_{a}\right)-\frac{\left|\mathbf{v}-\mathbf{U}_{a}\right|^{2}}{3} \mathbf{I}\right] f_{a} \\
& =\Pi_{a}-\frac{p_{a}}{3} \mathbf{I}-m_{a} n_{a} \mathbf{U}_{a} \mathbf{U}_{a} \\
\mathbf{F} & =\int d \mathbf{v} m_{a} \mathbf{v} \mathcal{C}_{a}\left[f_{a}\right] \\
F_{\|} & =\mathbf{b} \cdot \mathbf{F} \\
E_{\|} & =-\mathbf{b} \cdot \nabla \phi=-\nabla_{\|} \phi
\end{aligned}
$$

\section{Appendix B. Convergence of asymmetries}

To show the role of collisions in the formation of these asymmetries, we have run a simulation $(Z=10)$ for $1.5 \mathrm{~ms}$ without collisions (green) and re-started the simulation with the collisions on (yellow). The asymmetry is clearly shown to be attracted to the same state (red and yellow curves converge) despite the different history. These asymmetries are the consequence of the parallel friction force (PS regime) or of the parallel viscous force at lower collisionality (plateau regime), which are determined by plasma intrinsic properties (including the parallel flows). This is discussed in section 6 . Note that no source of impurities or perturbation that would break the system symmetry has been employed.

\section{REFERENCES}

Angioni, C., Casson, F. J., Mantica, P., Pütterich, T., Valisa, M., Belli, E. A., Bilato, R., Giroud, C. \& Helander, P. 2015 The impact of poloidal asymmetries on tungsten transport in the core of jet h-mode plasmas. Physics of Plasmas 22 (5), 055902.

Angioni, C \& Helander, P 2014 Neoclassical transport of heavy impurities with poloidally asymmetric density distribution in tokamaks. Plasma Physics and Controlled Fusion 56 (12), 124001.

Angioni, C., Mantica, P., Pütterich, T., Valisa, M., Baruzzo, M., Belli, E.A., Belo, P., Casson, F.J., Challis, C., Drewelow, P., Giroud, C., Hawkes, N., Hender, T.C., Hobirk, J., Koskela, T., Taroni, L. Lauro, Maggi, C.F., Mlynar, J., Odstrcil, T., Reinke, M.L., Romanelli, M. \& Contributors, Jet EFDA 2014 Tungsten transport in jet h-mode plasmas in hybrid scenario, experimental observations and modelling. Nuclear Fusion 54 (8), 083028. 
Verification of impurities and study of friction driven asymmetries
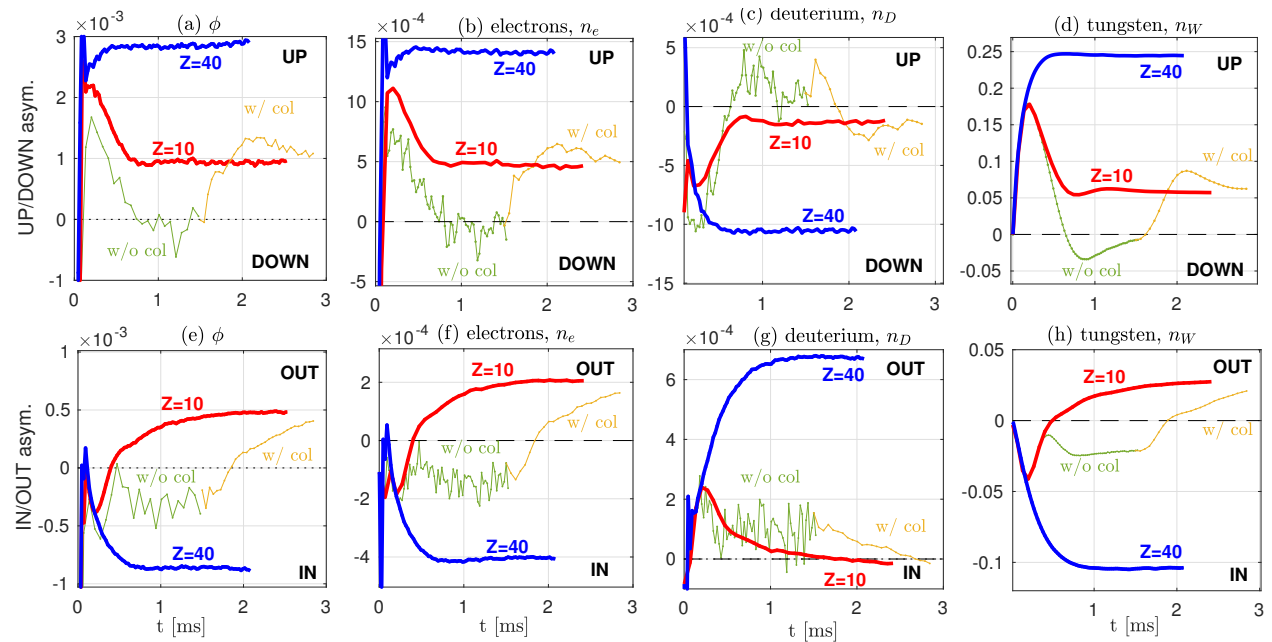

Figure 11. Time evolution of different fields asymmetries during the impure tungsten plasma simulations with $Z=10$ (red) and $Z=40$ (blue). Asymmetries are measured with equation (5.1) for the following fields: (a,e) the electrostatic potential, $(b, f)$ the electron density, $(\mathrm{c}, \mathrm{g})$ the deuterium density, and $(\mathrm{d}, \mathrm{h})$ the tungsten density. To illustrate the importance of collisions in the formation of asymmetries for $Z=10$ case, we also plot a simulation in green carried out with no collision until $t=1.5 \mathrm{~ms}$ and its continuation with collision switched on (at $t=1.5 \mathrm{~ms}$ ) in yellow. Diagnostics are measured at $\psi_{n}=0.45$ where the gradients are peaked. For this figure, $\mathcal{N}=1$ in equation $(5.1)$.

Belli, E A \& CAndy, J 2008 Kinetic calculation of neoclassical transport including selfconsistent electron and impurity dynamics. Plasma Physics and Controlled Fusion 50 (9), 095010.

Belli, E A \& CANDY, J 2012 Full linearized fokker-planck collisions in neoclassical transport simulations. Plasma Physics and Controlled Fusion 54 (1), 015015.

Brau, K., Suckewer, S. \& Wong, S.K. 1983 Vertical poloidal asymmetries of low-z element radiation in the pdx tokamak. Nuclear Fusion 23 (12), 1657-1668.

Breton, S., Casson, F. J., Bourdelle, C., Angioni, C., Belli, E., Camenen, Y., Citrin, J., Garbet, X., Sarazin, Y. \& Sertoli, M. 2018 High z neoclassical transport: Application and limitation of analytical formulae for modelling jet experimental parameters. Physics of Plasmas 25 (1), 012303.

Burrell, K.H. \& Wong, S.K. 1979 Self-consistent gyrokinetic modeling of neoclassical and turbulent impurity transport. Nuclear Fusion 19, 1571.

Chang, C.S. \& Hazeltine, R.D. 1980 Impurity transport in the collisional regime for large poloidal variations. Nuclear Fusion 20 (11), 1397-1405.

Chang, C. S. 1983 Enhancement of neoclassical transport coefficients by a poloidal electric field in tokamaks. The Physics of Fluids 26 (8), 2140-2149.

Chang, C. S. \& Hinton, F. L. 1982 Effect of finite aspect ratio on the neoclassical ion thermal conductivity in the banana regime. The Physics of Fluids 25 (9), 1493-1494.

Dominski, Julien, Ku, Seung-Hoe \& Chang, Choong-Seock 2018 Gyroaveraging operations using adaptive matrix operators. Physics of Plasmas 25 (5), 052304.

Estève, D., Sarazin, Y., Garbet, X., Grandgirard, V., Breton, S., Donnel, P., Asahi, Y., Bourdelle, C., Dif-Pradalier, G., Ehrlacher, C., Emeriau, C., Ghendrih, Ph., Gillot, C., Latu, G. \& Passeron, C. 2018 Self-consistent gyrokinetic modeling of neoclassical and turbulent impurity transport. Nuclear Fusion $\mathbf{5 8}$ (3), 036013.

FÜlöP, T. \& Helander, P. 1999 Nonlinear neoclassical transport in a rotating impure plasma with large gradients. Physics of Plasmas 6 (8), 3066-3075.

Guazzotto, L., Betti, R., Manickam, J. \& Kaye, S. 2004 Numerical study of tokamak equilibria with arbitrary flow. Physics of Plasmas 11 (2), 604-614.

Hager, Robert \& Chang, C. S. 2016 Gyrokinetic neoclassical study of the bootstrap current 
in the tokamak edge pedestal with fully non-linear coulomb collisions. Physics of Plasmas 23 (4), 042503.

Hager, R., Dominski, J. \& Chang, C.S. 2019 On the significance of the coulomb logarithm in benchmarks of neoclassical codes. accepted in Physics of Plasmas .

Hager, Robert, Yoon, E.S., Ku, S., D’Azevedo, E.F., Worley, P.H. \& Chang, C.S. 2016 A fully non-linear multi-species fokker planck landau collision operator for simulation of fusion plasma. Journal of Computational Physics 315, $644-660$.

Helander, P. 1998 Bifurcated neoclassical particle transport. Physics of Plasmas 5 (11), 39994004 .

Hinton, F. L. \& Hazeltine, R. D. 1976 Theory of plasma transport in toroidal confinement systems. Rev. Mod. Phys. 48, 239-308.

Hinton, F. L. \& Wong, S. K. 1985 Neoclassical ion transport in rotating axisymmetric plasmas. The Physics of Fluids 28 (10), 3082-3098.

Hirshman, S. P. \& SigmaR, D. J. 1976 Approximate fokker-planck collision operator for transport theory applications. The Physics of Fluids 19 (10), 1532-1540.

HubA, J D 2013 NRL PLASMA FORMULARY Supported by The Office of Naval Research. Washington, DC: Naval Research Laboratory.

Kim, Kyuho, Kwon, Jae-Min, Chang, C. S., Seo, Janghoon, Ku, S. \& Choe, W. 2017 Full-f xgc1 gyrokinetic study of improved ion energy confinement from impurity stabilization of itg turbulence. Physics of Plasmas 24 (6), 062302.

Köchl, F, Loarte, A, de la Luna, E, Parail, V, Corrigan, G, Harting, D, Nunes, I, Reux, C, Rimini, F G, Polevoi, A, Romanelli, M \& Contributors, Jet 2018 $\mathrm{W}$ transport and accumulation control in the termination phase of jet h-mode discharges and implications for iter. Plasma Physics and Controlled Fusion 60 (7), 074008.

Ku, S., Chang, C. S., Hager, R., Churchill, R. M., Tynan, G. R., Cziegler, I., Greenwald, M., Hughes, J., Parker, S. E., Adams, M. F., D’Azevedo, E. \& Worley, P. 2018 A fast low-to-high confinement mode bifurcation dynamics in the boundary-plasma gyrokinetic code xgc1. Physics of Plasmas 25 (5), 056107.

Ku, S., Hager, R., Chang, C.S., Kwon, J.M. \& Parker, S.E. 2016 A new hybridlagrangian numerical scheme for gyrokinetic simulation of tokamak edge plasma. Journal of Computational Physics 315, 467 - 475.

Litaudon, X. \& ET AL. 2017 Overview of the jet results in support to iter. Nuclear Fusion 57 (10), 102001.

Loarte, A., Reinke, M. L., Polevoi, A. R., Hosokawa, M., Chilenski, M., Howard, N., Hubbard, A., Hughes, J. W., Rice, J. E., Walk, J., Köchl, F., Pütterich, T., Dux, R. \& Zhogolev, V. E. 2015 Tungsten impurity transport experiments in alcator c-mod to address high priority research and development for iter. Physics of Plasmas $22(5), 056117$.

Parker, S.E., Procassini, R.J., Birdsall, C.K. \& Cohen, B.I. 1993 A suitable boundary condition for bounded plasma simulation without sheath resolution. Journal of Computational Physics 104 (1), 41 - 49.

Reinke, M. L. 2011 Experimental tests of parallel impurity transport theory in tokamak plasmas. PhD thesis, Massachusetts Institute of Technologies.

Reinke, M. L., Hutchinson, I. H., Rice, J. E., Greenwald, M., Howard, N. T., Hubbard, A., Hughes, J. W., Terry, J. L. \& Wolfe, S. M. 2013 Parallel transport studies of high-z impurities in the core of alcator c-mod plasmas. Physics of Plasmas 20 (5), 056109.

Romanelli, M \& Ottaviani, M 1998 Effects of density asymmetries on heavy impurity transport in a rotating tokamak plasma. Plasma Physics and Controlled Fusion 40 (10), 1767.

Sauter, O., Angioni, C. \& Lin-Liu, Y. R. 1999 Neoclassical conductivity and bootstrap current formulas for general axisymmetric equilibria and arbitrary collisionality regime. Physics of Plasmas 6 (7), 2834-2839.

Stotler, D.P., Lang, J., Chang, C.S., Churchill, R.M. \& Ku, S. 2017 Neutral recycling effects on ITG turbulence. Nuclear Fusion 57 (8), 086028.

Tendler, M. 1981 Impurity transport in a rotating toroidal plasma with cold-gas mantle system. International Atomic Energy Agency (IAEA). 